18,12

\title{
Модельный подход к описанию свойств графана: аналитические результаты
}

\author{
() С.Ю. Давыдов
}

Физико-технический институт им. А.Ф. Иофффе РАН,

Санкт-Петербург, Россия

E-mail: Sergei_Davydov@mail.ru

Поступила в Редакцию 25 июля 2020 г.

В окончательной редакции 25 июля 2020 г.

Принята к публикации 14 августа 2020 г.

Методом функций Грина в приближении сильной связи получены аналитические выражения для дисперсии электронных зон. Предложена параболическая аппроксимация спектра, в рамках которой определены значения эффективной массы носителей и квантовой емкости. С использованием моделей Костера-Слэтера и Халдейна-Андерсона рассмотрена задача о локальных состояниях дефектов. Приведены аналитические оценки характерных фононных частот и упругих постоянных. Полученные результаты сопоставлены с данными расчетов других авторов.

Ключевые слова: эффективная масса, квантовая емкость, моно- и бивакансия, фононы, упругие постоянные.

DOI: $10.21883 /$ FTT.2020.12.50224.158

\section{1. Введение}

Графан - гидрогенизированный графен, все свободные $p$-орбитали которого пассивированы атомами водорода, расположенными поочередно по разным сторонам графенового листа, - был придуман теоретиками [1] и вскоре синтезирован [2]. В расчетах $[1,3,4]$ показано, что графан является полупроводником с широкой запрещенной зоной $E_{g} \sim 3.5-6 \mathrm{eV}$. К этим расчетам следует добавить работы [5-8], посвященные свойствам частично гидрогенезированного графена (в частности, наведенными водородными вакансиями магнитным состояниям $[3,5,6,8]$ и сверхпроводимостью [8]). В статье [9] рассмотрена возможность использования дефектного графана для квантовых вычислений. Таким образом, интерес к графану не затухает.

Во всех цитированных работах (за исключением [8,9]) для расчетов используются различные варианты DFT (density functional theory). C точки зрения использования графана в реальных приборных структурах необходима упрощенная теория (см., например, [9]), которая, однако, адекватно описывает характерные черты графана (см. [8]). Здесь мы предложим достаточно простую модель электронного спектра графана, используя при этом результаты работы [10], посвященной графеноподобным соединениям. В рамках такой модели удается получить аналитические выражения для эффективной массы носителей и квантовой емкости идеального графана и рассмотреть задачу о лежащих в запрещенной зоне локальных дефектных уровнях. Предложен также простой подход к оценкам характерных фононных частот и упругих постоянных.

\section{2. Электронный спектр графана}

\section{1. Модель}

Начнем с общей задачи о графаноподобном бинарном соединении, образованном лежащими в узлах гексагональной двумерной решетки атомами А и В, взаимодействующими как между собой, так и с атомами водорода. Рассмотрим для начала асимметричный димер, состоящий из атома $\mathrm{A}(\mathrm{B})$ и атома $\mathrm{H}$, взаимодействие между которыми описывается матричным элементом $t_{a(b) h}$. Соответствующие функции Грина такого димера имеют вид

$$
\begin{gathered}
G^{\mathrm{A}(\mathrm{B})}(\omega)=\frac{g^{\mathrm{A}(\mathrm{B})}(\omega)}{1-t_{a(b) h}^{2} g^{\mathrm{A}(\mathrm{B})}(\omega) g^{\mathrm{H}}(\omega)}, \\
G^{\mathrm{H}}(\omega)=\frac{g^{\mathrm{H}}(\omega)}{1-t_{a(b) h}^{2} g^{\mathrm{A}(\mathrm{B})}(\omega) g^{\mathrm{H}}(\omega)} .
\end{gathered}
$$

Здесь $\omega-$ энергетическая переменная, $g^{\mathrm{A}(\mathrm{B})}(\omega)=$ $=\left(\omega-\varepsilon_{a(b)}+i 0^{+}\right)^{-1}-$ функция Грина изолированного атома $\mathrm{A}(\mathrm{B}), g^{\mathrm{H}}(\omega)=\left(\omega-\varepsilon_{h}+i 0^{+}\right)^{-1}-$ функции Грина изолированного атома водорода, $\varepsilon_{i}(i=a, b, h)-$ энергии уровней атомов $\mathrm{A}, \mathrm{B}, \mathrm{H}$.

Теперь построим из этих димеров гексагональную двумерную решетку, включив взаимодействие $t_{a b}$ между ближайшими А и В компонентами „антипараллельных“ димеров. Тогда, обобщая результаты работы [10], функция Грина для графаноподобного бинарного соединения может быть представлена в виде

$$
G^{\mathrm{AA}(\mathrm{BB})}(\omega, \mathbf{k})=\frac{G^{\mathrm{A}(\mathrm{B})}(\omega)}{1-t_{a b}^{2} G^{\mathrm{A}}(\omega) G^{\mathrm{B}}(\omega) f^{2}(\mathbf{k})},
$$




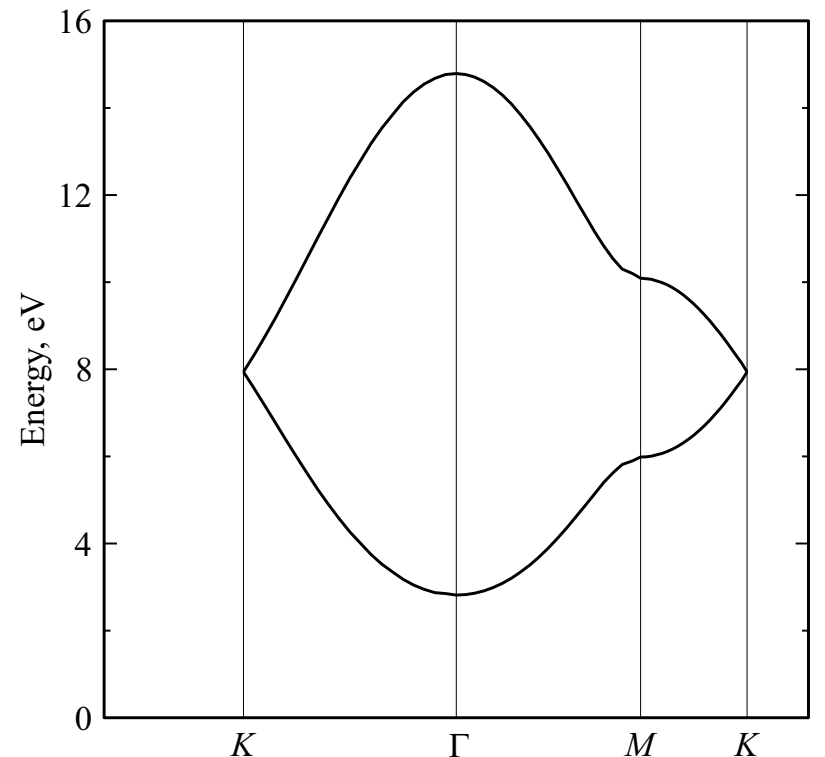

Рис. 1. Дисперсия электронов в графане, вычисленная по формулам (4) и (5). На рисунке представлены только зоны $E_{1}(\mathbf{k})$ и $E_{C}(\mathbf{k})$, отвечающие положительной энергии.

где

$$
f(\mathbf{k})=\sqrt{3+2 \cos \left(k_{x} a \sqrt{3}\right)+4 \cos \left(k_{x} a \sqrt{3} / 2\right) \cos \left(3 k_{y} a / 2\right)}
$$

(ось $x$ направлена вдоль зигзагообразной кромки), $a=1.542 \AA-$ расстояние между ближайшими атомами $\mathrm{A}$ и $\mathrm{B}, \mathbf{k}=\left(k_{x}, k_{y}\right)$ - волновой вектор для движения электрона в плоскости графенового листа. Соответствующий электронный спектр определяется из уравнения $\operatorname{Re} D(\omega, \mathbf{k})=0$, где

$$
\begin{aligned}
D(\omega, \mathbf{k})= & {\left[1-t_{a h}^{2} g^{\mathrm{A}}(\omega) g^{\mathrm{H}}(\omega)\right]\left[1-t_{b h}^{2} g^{\mathrm{B}}(\omega) g^{\mathrm{H}}(\omega)\right] } \\
& -t_{a b}^{2} g^{\mathrm{A}}(\omega) g^{\mathrm{B}}(\omega) f^{2}(\mathbf{k}) .
\end{aligned}
$$

Переходя к графану, для которого атомы А и В являются атомами углерода и характеризуются энергией $p$-орбитали $\varepsilon_{c}$, а атомы водорода Н - энергией $s$-орбитали $\varepsilon_{h}$, и положив $\varepsilon_{c}=-\varepsilon_{h}=\varepsilon, t_{a(b) h}=t_{\perp}, t_{a b}=t$, получим электронный спектр графана в виде

$$
\begin{gathered}
E_{1}(\mathbf{k})=\frac{1}{2}\left(\sqrt{[|\varepsilon|+t f(\mathbf{k})]^{2}+4 t_{\perp}^{2}}+t f(\mathbf{k})\right)=-E_{4}(\mathbf{k}) \\
\begin{aligned}
E_{2}(\mathbf{k})=E_{C}(\mathbf{k}) & =\frac{1}{2}\left(\sqrt{[|\varepsilon|-t f(\mathbf{k})]^{2}+4 t_{\perp}^{2}}-t f(\mathbf{k})\right) \\
& =-E_{3}(\mathbf{k})=-E_{V}(\mathbf{k})
\end{aligned}
\end{gathered}
$$

где индексы 1-4 нумеруют зоны, расположенные по убыванию их энергии, а индексы $C$ и $V$ отвечают соответственно зоне проводимости и валентной зоне. Характерные энергетические интервалы равны

$$
\Delta_{\mathrm{K}}=E_{C}(\mathrm{~K})-E_{V}(\mathbf{K})=r_{0},
$$

$$
\begin{gathered}
\Delta_{\mathrm{M}}=E_{C}(\mathrm{M})-E_{V}(\mathrm{M})=r_{2-}-2 t, \\
\Delta_{\Gamma}=E_{g}=E_{C}(\Gamma)-E_{V}(\Gamma)=r_{3-}-3 t,
\end{gathered}
$$

где $\mathrm{K}, \mathrm{M}, \Gamma-$ точки зоны Бриллюэна графана, $r_{0}=\sqrt{\varepsilon^{2}+4 t_{\perp}^{2}}$

$$
r_{2 \pm}=\sqrt{(\varepsilon \pm 2 t)^{2}+4 t_{\perp}^{2}}, \quad r_{3 \pm}=\sqrt{(\varepsilon \pm 3 t)^{2}+4 t_{\perp}^{2}} .
$$

Для определения параметров модели воспользуемся результатами работы [4] (см. GW-вариант расчета), согласно которым $\Delta_{\mathrm{K}}=15.9 \mathrm{eV}, \Delta_{\mathrm{M}}=13.7 \mathrm{eV}$, $E_{g}=5.6 \mathrm{eV}$. Тогда, полагая $t=2.8 \mathrm{eV}[9,11]$, приближенно найдем: $t_{\perp}=7 \mathrm{eV}$ и $|\varepsilon|=7.5 \mathrm{eV}$. Используя эти значения параметров, получим зоны, представленные на рис. 1.

\section{2. Аппроксимация зонного спектра}

Исходя из выражения (5), для приведенных эффективных масс электронов $m_{e}$ и дырок $m_{h}=-m_{e}$ имеем

$$
m_{e}=\frac{4 \hbar^{2}}{3 a^{2} t}\left(1-\frac{3 t-|\varepsilon|}{r_{3}-}\right)^{-1},
$$

так что $m_{e}^{*}=m_{e} / m_{0} \approx 1.9$, где $m_{0}-$ масса свободного электрона.

Для решения задачи о дефектах нужно найти аналитическое выражение для зонных плотностей состояний $\rho_{C}(\omega)$ и $\rho_{V}(\omega)$. Поэтому аппроксимируем дисперсию зоны проводимости и валентной зоны выражением

$$
E_{C}(\mathbf{k})=-E_{V}(\mathbf{k})=\frac{E_{g}}{2}+\frac{\hbar^{2} k^{2}}{2 m_{e}},
$$

где $k=\sqrt{k_{x}^{2}+k_{e}^{2}}$. Для зон $E_{1}(\mathbf{k})=-E_{4}(\mathbf{k})$ запишем

$$
E_{1}(\mathbf{k})=\frac{E_{g}}{2}-\frac{\hbar^{2} k^{2}}{2 m_{1}}
$$

где

$$
m_{1}=\frac{4 \hbar^{2}}{3 a^{2} t}\left(1+\frac{3 t+|\varepsilon|}{r_{3+}}\right)^{-1},
$$

откуда получаем $m_{1}^{*}=m_{1} / m_{0} \approx 1.2$. Графики функций (8) и (9) представлены на рис. 2 (отмечены темными кружками) в сопоставлении с зависимостями $E_{1}(\mathbf{k})$ и $E_{C}(\mathbf{k})$, рассчитанными по формулам (4) и (5) (жирные линии). Аппроксимацию следует признать вполне удовлетворительной, имея в виду, что в дальнейшем нас будут интересовать интегральные характеристики. Отметим, однако, что, согласно (4) и (5), $E_{1}(\mathbf{k}) \propto|\mathbf{k}|$ при $\mathbf{k} \rightarrow \mathrm{K}$ (как и в случае листа графена), тогда как согласно аппроксимации $E_{1}(\mathbf{k}) \propto k^{2}$.

В двумерных системах спектрам (8) и (9) в определенном энергетическом интервале отвечают плотности состояний $\rho(\omega)=$ const (модель типа „пьедестал“). Тогда положим $\rho_{C}(\omega)=\rho_{V}(\omega)=2 / W_{C}$ при $E_{C}(\Gamma) \leq|\omega| \leq E_{C}(\mathrm{~K})$, где $W_{C}=W_{V}=\left(3 t+r_{0}-r_{3-}\right) / 2=5.1 \mathrm{eV}$ - ширина 


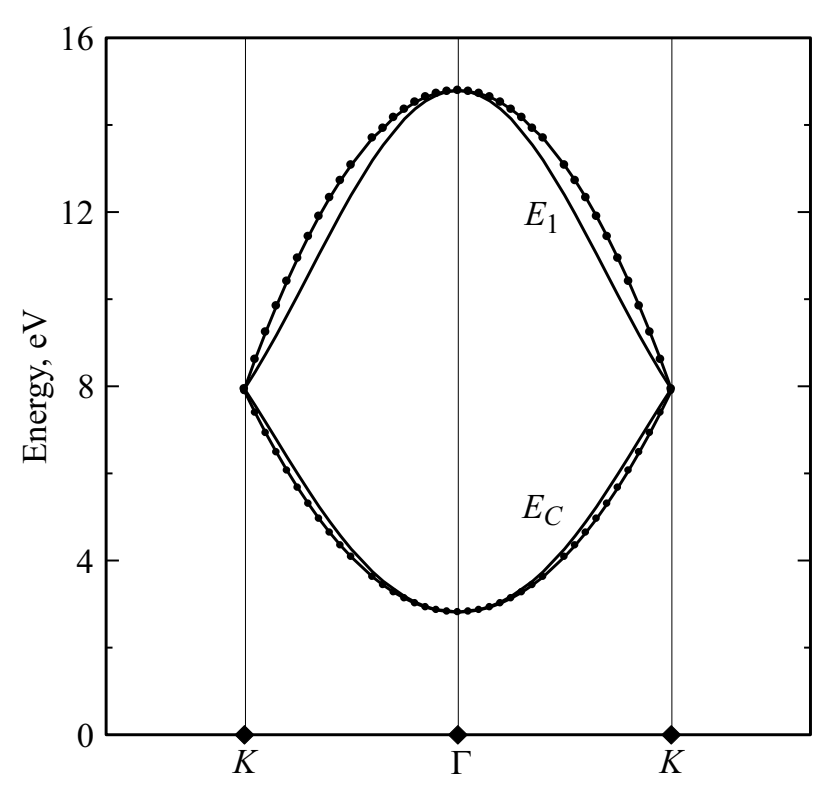

Рис. 2. Параболическая аппроксимация (линии с жирными точками) зон $E_{1}(\mathbf{k})$ и $E_{C}(\mathbf{k})$ (жирные линии), рассчитанная по формулам (8) и (9).

зон проводимости и валентной, множитель 2 соответствует числу заполнения зон электронами. Аналогичным образом плотности состояний для зон $E_{1}(\mathbf{k})=-E_{4}(\mathbf{k})$ при $E_{1}(\mathrm{~K})=E_{C}(\mathrm{~K}) \leq|\omega| \leq E_{1}(\Gamma)$ могут быть представлены в виде $\rho_{1}(\omega)=\rho_{4}(\omega)=2 / W_{1}$, $W_{1}=W_{4}=\left(3 t+r_{3+}-r_{0}\right) / 2=6.9 \mathrm{eV}$. Полная плотность состояний графана равна $\rho(\omega)=\rho_{C}(\omega)+\rho_{1}(\omega)$.

\section{3. Квантовая емкость}

Понятие квантовая емкость $C_{Q}$ было введено в работах $[12,13]$. Применяя к графану выражение для $C_{Q}$, предложенное в [14] для двумерной наноструктуры, запишем:

$$
C_{Q}=\left(e^{2} / 4 T S\right) \int_{0}^{\infty} \rho(\omega) A\left(\omega, V^{*}\right) d \omega
$$

$$
A\left(\omega, V^{*}\right)=\operatorname{sch}^{2}\left[\left(\omega-V^{*}\right) / 2 T\right]+\operatorname{sch}^{2}\left[\left(\omega+V^{*}\right) / 2 T\right],
$$

где $V^{*}=e V_{e l}$ - сдвиг электронных состояний в электростатическом поле, $V_{e l}-$ внешний электростатический потенциал, $e-$ величина заряда электрона, $T$ температура в энергетических единицах, $S$ - площадь графенового листа, приходящаяся на один атом углерода; считаем, что при $V^{*}=0$ химический потенциал совпадает с центром запрещенной зоны графана. Вводя безразмерные параметры

$$
\begin{gathered}
x=\omega / 2 T, \quad v^{*}=V^{*} / 2 T, \quad e_{g}=E_{g} / 2 T, \\
e_{1}=E_{1}(\Gamma) / 2 T \quad \text { и } e_{2}=E_{C}(\mathrm{~K}) / 2 T=E_{1}(\mathrm{~K}) / 2 T,
\end{gathered}
$$

из (11) получим

$$
\begin{gathered}
C_{Q}\left(V^{*}\right)=\left(e^{2} / 2 S\right) I, \quad I=\left(I_{1}+I_{2}\right), \\
I_{1}=\frac{2}{W_{1}} \int_{e_{2}}^{e_{1}} A\left(x, v^{*}\right) d x, \quad I_{2}=\frac{2}{W_{C}} \int_{e_{g} / 2}^{e_{2}} A\left(x, v^{*}\right) d x,
\end{gathered}
$$

Легко показать, что

$$
\begin{gathered}
I_{1}=\left(2 / W_{1}\right)\left[\operatorname{th}\left(e_{1}+v^{*}\right)+\operatorname{th}\left(e_{1}-v^{*}\right)\right. \\
\left.-\operatorname{th}\left(e_{2}+v^{*}\right)-\operatorname{th}\left(e_{2}-v^{*}\right)\right], \\
I_{2}=\left(2 / W_{C}\right)\left[\operatorname{th}\left(e_{2}+v^{*}\right)+\operatorname{th}\left(e_{2}-v^{*}\right)\right. \\
\left.-\operatorname{th}\left(e_{g} / 2+v^{*}\right)-\operatorname{th}\left(e_{g} / 2-v^{*}\right)\right] .
\end{gathered}
$$

Так как при комнатной температуре $e_{1} \gg 1$ и $e_{g} / 2 \gg 1$, то при $v^{*} \ll e_{g} / 2$ получим

$$
\begin{gathered}
I_{1} \approx \frac{8 \operatorname{ch}\left(2 v^{*}\right)}{W_{1}}\left[\exp \left(-2 e_{2}\right)-\exp \left(-2 e_{1}\right)\right], \\
I_{2} \approx \frac{8 \operatorname{ch}\left(2 v^{*}\right)}{W_{C}}\left[\exp \left(-e_{g}\right)-\exp \left(-2 e_{2}\right)\right] .
\end{gathered}
$$

Считая для простоты, что $W_{1} \approx W_{C}=W$, окончательно находим

$$
I \approx \frac{8 \operatorname{ch}\left(V^{*} / T\right)}{W} \exp \left(-E_{g} / 2 T\right) .
$$

Квантовая емкость $C_{Q}^{0} \equiv C_{Q}(0) \approx(16 / 3 \sqrt{3})\left(e^{2} / W a^{2}\right)$ $\times \exp \left(-E_{g} / 2 T\right)$, так что для комнатной температуры получаем $C_{Q}^{0}=0$ (для графена $C_{Q}^{0}=1.6 \mu \mathrm{F} / \mathrm{cm}^{2}$ [15]); $C_{Q}\left(E_{g} / 2\right) \approx(8 / 3 \sqrt{3})\left(e^{2} / W a^{2}\right)=0.2 \mathrm{mF} / \mathrm{cm}^{2}$.

\section{3. Локальные состояния дефектов}

\section{1. Модель Костера-Слэтера}

Перейдем теперь к задаче о точечном дефекте в графане, воспользовавшись моделью КостераСлэтера [16-18], удобной для описания вакансий и примесей внедрения и применявшейся нами для листа [19] и наноленты [20] графена.

Пусть возмущение, вносимое дефектом, сосредоточено в одном узле графана и равно $V$. Тогда, как показано в [19], плотность состояний на дефекте $\rho_{d}(\omega)$ можно приближенно представить в виде

$$
\begin{gathered}
\rho_{d}(\omega) \approx \frac{\rho(\omega)}{[1-V L(\omega)]^{2}+[\pi V \rho(\omega)]^{2}}, \\
L(\omega)=P \int_{-\infty}^{\infty} \frac{\rho\left(\omega^{\prime}\right) d \omega^{\prime}}{\omega-\omega^{\prime}},
\end{gathered}
$$




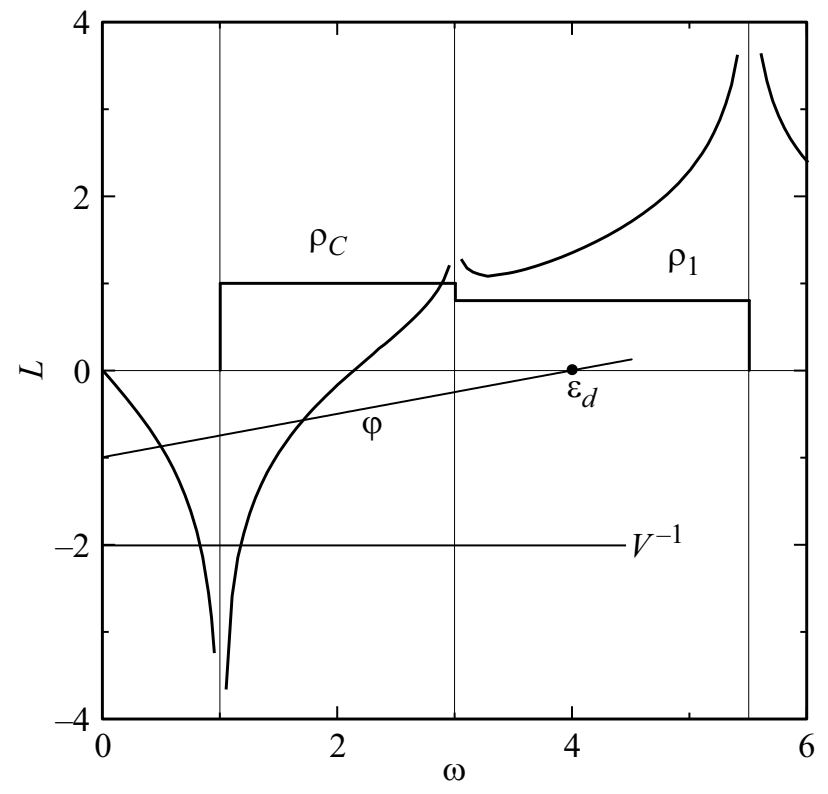

Рис. 3. Функция $L(\omega)$ для плотностей состояний $\rho_{C}(\omega)$ и $\rho_{1}(\omega)$ (жирные линии). Вертикальными штриховыми линиями изображены асимптоты, соответствующие краям зон $E_{C}(\mathbf{k})$ и $E_{1}(\mathbf{k})$. Тонкими линиями изображены функции $V^{-1}$ (модель Костера-Слэтера) и $\varphi(\omega)=\left(\omega-\varepsilon_{d}\right) V_{d}^{2}$ (модель ХалдейнаАндерсона); точки пересечения этих линий с $L(\omega)$ определяют положение уровней дефекта. На рисунке представлена только область положительных энергий, так как $\rho_{C, 1}(\omega)=\rho_{C, 1}(-\omega)$, $L(\omega)=-L(-\omega)$. Энергетические величины измерены в ед. $t$, функции $L(\omega)$ и $\rho_{C, 1}(\omega)-$ в ед. $t^{-1}$.

где $P-$ символ главного значения интеграла. Легко показать, что

$$
\begin{aligned}
L(\omega)= & \frac{2}{W_{1}} \ln \left|\frac{\left[E_{C}(\mathrm{~K})-\omega\right]\left[E_{1}(\Gamma)+\omega\right]}{\left[E_{C}(\mathrm{~K})+\omega\right]\left[E_{1}(\Gamma)-\omega\right]}\right| \\
& +\frac{2}{W} \ln \left|\frac{\left[E_{C}(\mathrm{~K})+\omega\right]\left[E_{g} / 2-\omega\right]}{\left[E_{C}(\mathrm{~K})-\omega\right]\left[E_{g} / 2+\omega\right]}\right| .
\end{aligned}
$$

Функция $L(\omega)$ представлена на рис. 3. Энергии уровней дефекта определяется корнями уравнения

$$
L(\omega)=V^{-1}
$$

Далее мы будем рассматривать только локальные состояния $\omega_{l}$, лежащие в запрещенной зоне $\left(-E_{g} / 2 \leq \omega_{l} \leq E_{g} / 2\right)$. Плотности состояний таких уровней $\rho_{l}(\omega)$, числа заполнения при нулевой температуре $n_{l}$ и фактор заполнения $v_{1}$ определяются соответственно выражениям

$$
\begin{gathered}
\rho_{l}(\omega)=v_{l} \delta\left(\omega-\omega_{l}\right), \quad n_{l}=v_{l} \Theta\left(E_{F}-\omega_{l}\right), \\
v_{l} \approx\left|V^{2} d L(\omega) / d \omega\right|_{\omega_{l}}^{-1},
\end{gathered}
$$

где $\Theta(.$.$) - функция Хэвисайда, E_{F}-$ уровень Ферми. Из (18) получаем

$$
\begin{aligned}
\frac{d L(\omega)}{d \omega}= & -\frac{2 E_{g}}{W_{C}\left[\left(E_{g} / 2\right)^{2}-\omega^{2}\right]}+\frac{4 E_{1}(\Gamma)}{W_{1}\left[E_{1}^{2}(\Gamma)-\omega^{2}\right]} \\
& +\frac{4 E_{C}(\mathrm{~K})}{E_{C}^{2}(\mathrm{~K})-\omega^{2}}\left(\frac{1}{W_{C}}-\frac{1}{W_{1}}\right) .
\end{aligned}
$$

Так как в данной работе мы используем достаточно простые модели и не стремимся получить строгие результаты, будем считать, что $E_{1}(\Gamma) \gg E_{g} / 2$ и вновь полагать $W_{1} \approx W_{C}=W$. Тогда для области запрещенной зоны получим

$$
\begin{gathered}
L(\omega) \approx \frac{2}{W} \ln \left(\frac{E_{g} / 2-\omega}{E_{g} / 2+\omega}\right), \\
\frac{d L(\omega)}{d \omega} \approx-\frac{2 E_{g}}{W\left[\left(E_{g} / 2\right)^{2}-\omega^{2}\right]} .
\end{gathered}
$$

Рассмотрим два предела: $|V| \ll E_{g} / 2, W \quad$ и $|V| \gg E_{g} / 2, W$. В первом случае для $V>0$ и $V<0$ получаем соответственно

$$
\omega_{l} \approx \mp\left(E_{g} / 2\right)[1-2 \exp (-W / 2|V|)]
$$

и

$$
v_{l} \approx\left(W E_{g} / 2 V^{2}\right) \exp (-W / 2|V|) .
$$

Во втором случае имеем $\omega_{l} \approx-W E_{g} / 2 V$ и $v_{l} \approx W E_{g} / 2 V^{2}$. Таким образом, при $|V| \ll E_{g} / 2, W$ рост $|V|$ приводит к сдвигу уровней к границам запрещенной зоны и увеличению фактора заполнения $v_{l}$, во втором случае - к сдвигу уровня к центру зоны и уменьшению $v_{l}$.

В общем случае характер зависимостей $\left|\omega_{l}\right|$ от $|V|$ легко понять, исходя из уравнения (19) и рис 3: с ростом $|V|$ функция $|L(\omega)|$ должна уменьшаться, что происходит при сдвиге $\left|\omega_{l}\right|$ к центру запрещенной зоны. $\mathrm{C}$ функцией $v_{l}(V)$ дело обстоит сложнее. Действительно, с ростом $|V|$ величина $|d L(\omega) / d \omega|_{\omega_{l}=0}$ уменьшается. При малых потенциалах возмущения уменьшение $|d L(\omega) / d \omega|_{\omega_{l}=0}$ превышает увеличение $V^{2}$, так что $v_{l}$ растет. При больших $|V|$ имеем обратную ситуацию: с ростом $|V|$ величина $v_{l}$ убывает. Необходимо, однако, учесть следующее обстоятельство. В настоящей работе для плотности состояний принята модель типа „Пьедестал“ или ступеньки (22). Именно скачки $\rho(\omega)$, характерные для таких моделей, приводят к расходимостям функции $L(\omega)$, в результате чего мы получаем локальные уровни при сколь угодно малом значении возмущения $|V|$. Если же скачки $\rho(\omega)$ отсутствуют, то при $\omega= \pm E_{g} / 2, \pm E_{C}(\mathrm{~K}), \pm E_{1}(\Gamma)$ функция $L(\omega)$ имеет в этих точках экстремальные, но конечные значения $L_{\text {extr. }}$ При этом, когда $|V|<V_{\min }=\left|L_{\text {extr }}^{-1}\left( \pm E_{g} / 2\right)\right|$ локальные состояния в запрещенной зоне отсутствуют. Таким образом, восходящая ветвь функции $v_{l}(|V|)$ является артефактом использованной модели. 
Предел $|V| \rightarrow \infty$, когда $\omega_{l} \rightarrow 0$ и $v_{l} \rightarrow 0$, соответствует наличию вакансии в матрице. Для графана такая вакансия отвечает удалению одного димера, энергия $s p$ орбитали которого $\varepsilon_{\operatorname{dim}}=\left(\varepsilon_{c}+\varepsilon_{h}\right) / 2=0$. Водородным и углеродным вакансиям, а также примесям замещения соответствуют конечные значения потенциала $V$. При этом возникает вопрос о величине и знаке такого возмущения. (Именно эта особенность модели Костера-Слэтера затрудняет ее использование для конкретных дефектных структур). Согласно результатам расчета [4], энергетический уровень водородной вакансии $\omega_{l}^{h}$ расположен на $2 \mathrm{eV}$ выше потолка валентной зоны, что в нашей системе отсчета энергии соответствует $\omega_{l}^{h}=-0.8 \mathrm{eV}$. Подставляя это значение в (22), получим $V \approx 5 \mathrm{eV}$ и $\nu_{l} \approx 0.3$.

\section{2. Модель Халдейна-Андерсона}

Рассмотрим теперь модель Халдейна-Андерсона [21], которая использовалась для описания примесей в полупроводниках и для чужеродных атомов, на них адсорбированных [22]. Без учета кулоновского отталкивания, плотность состояний на точечном дефекте в графане равна

$$
\rho_{d}(\omega)=\frac{2}{\pi} \frac{\Gamma(\omega)}{\left(\omega-\varepsilon_{d}-\Lambda(\omega)\right)^{2}+\Gamma(\omega)^{2}},
$$

где полуширина и энергетический сдвиг уровня дефекта $\varepsilon_{d}$ равны соответственно $\Gamma(\omega)=\pi V_{d}^{2} \rho(\omega)$ и $\Lambda(\omega)=V_{d}^{2} L(\omega), V_{d}$ - потенциал взаимодействия дефекта с матрицей-графаном. Энергии примесных состояний определяются корнями уравнения

$$
\omega-\varepsilon_{d}-\Lambda(\omega)=0
$$

Плотности состояний лежащих в запрещенной зоне локальных уровней и их числа заполнения определяются выражениями (14), факторы заполнения, однако, равны $v_{l}=|1-d \Lambda(\omega) / d \omega|_{\omega_{l}}^{-1}$, откуда, вновь прибегая к упрощениям (16), получаем

$$
v_{l}=\left(1+\frac{2 E_{g} V_{d}^{2}}{W\left[\left(E_{g} / 2\right)^{2}-\omega_{l}^{2}\right]}\right)^{-1} .
$$

Таким образом, по сравнению с моделью КостераСлэтера, модель Халдейна-Андерсона, во-первых, содержит дополнительный параметр $\varepsilon_{d}$. Во-вторых, потенциал $V_{d}$ кардинальным образом отличается от возмущения $V$ : первый отвечает туннелированию электрона между уровнем дефекта и зонными состояниями матрицы, тогда как второй описывает возмущение, вызванное заменой атома матрицы на чужеродный атом.

Рассмотрим два частных случая: $\left|\varepsilon_{d}\right| \ll E_{g} / 2, W$ и $\left|\varepsilon_{d}\right| \gg E_{g} / 2, W$.

В первом случае получим $\omega_{l} \approx v_{l} \varepsilon_{d}$, где $v_{l} \approx\left(1+8 V_{d}^{2} / W E_{g}\right)^{-1} \sim 1$. Во втором случае при $\varepsilon_{d}>0$ и $\varepsilon_{d}<0$ имеем соответственно

$$
\omega_{l} \approx \pm\left(E_{g} / 2\right)\left[1-2 \exp \left(-\left|\varepsilon_{d}\right| W / 2 V_{d}^{2}\right)\right]
$$

и

$$
v_{l} \approx\left(W E_{g} / 8 V_{d}^{2}\right) \exp \left(-\left|\varepsilon_{d}\right| W / 2 V_{d}^{2}\right) .
$$

В общем случае, качественный характер зависимостей $\omega_{l}\left(\varepsilon_{d}\right)$ и $\omega_{l}\left(V_{d}\right)$ можно усмотреть из рис. 3 , представив уравнение $(24)$ в виде $\varphi(\omega) \equiv\left(\omega-\varepsilon_{d}\right) / V_{d}^{2}=L(\omega)$ : сдвиг $\varepsilon_{d}$ в направлении больших энергий приводит к аналогичному сдвигу уровней $\omega_{l}$; увеличение $V_{d}$ смещает уровни $\omega_{l}$ к центру запрещенной зоны. Сoгласно (19), максимальное значение фактора заполнения $\left(v_{l}\right)_{\max }=\left(1+8 V_{d}^{2} / W E_{g}\right)^{-1}$ достигается при $\omega_{l}=0$, тогда как минимальное значение $\left(v_{l}\right)_{\min }=0$ отвечает $\omega_{l}= \pm E_{g} / 2$.

Насколько известно автору, до сих пор модель Халдейна-Андерсона не применялась для описания вакансий. Так как эта модель бесструктурна (с кристаллографической точки зрения), то в случае графана водородную вакансию можно представить в виде взаимодействующего с матрицей одиночного атома углерода. Вновь воспользовавшись значением энергетического уровня водородной моновакансии $\omega_{l}^{h}=-0.8 \mathrm{eV}$ [4], получим, согласно (25), фактор заполнения $v_{l}^{h} \approx 0.29$ и $\varepsilon_{d} \approx-2.7 \mathrm{eV}$, где мы положили $V_{d}=t$ (по поводу последнего равенства см. [23]).

Рассмотрим теперь водородную бивакансию, которую можно представить как взаимодействующий с графаном C-C-димер. Функция Грина такого димера равна $G_{\operatorname{dim}}(\omega)=g_{d}(\omega)\left[1-g_{d}^{2}(\omega)\right]^{-1}$, где функция Грина моновакансии $g_{d}^{-1}(\omega)=\omega-\varepsilon_{d}-\Lambda(\omega)+i \Gamma(\omega)$. Согласно [24], соответствующая плотность состояний углеродного димера равна

$$
\begin{gathered}
\rho_{\operatorname{dim}}(\omega)=\frac{1}{2}\left[\rho_{-}(\omega)+\rho_{+}(\omega)\right], \\
\rho_{ \pm}=\frac{2}{\pi} \frac{\Gamma(\omega)}{\left[\omega-\varepsilon_{d}-\Lambda(\omega) \pm t\right]^{2}+\Gamma^{2}(\omega)},
\end{gathered}
$$

а энергетические уровни бивакансии определяются корнями уравнений

$$
\omega-\varepsilon_{d}-\Lambda(\omega) \pm t=0
$$

Для локальных уровней $\omega_{l+}^{h}$, лежащих в запрещенной зоне получим $\omega_{l-}^{h} \approx-1.6 \mathrm{eV},\left(v_{l-}^{h} \approx 0.23\right)$ и $\omega_{l+}^{h} \approx 0$, $\left(v_{l+}^{h} \approx 0.31\right)$. Таким образом, уровень моновакансии $\omega_{l}^{h}=-0.8 \mathrm{eV}$ [4] расщепляется на два уровня, являющихся аналогами связывающего и антисвязывающего состояний двухатомной молекулы. В [9] задача о водородной бивакансии в графане рассматривалась с учетом внутриатомного кулоновского отталкивания $U=6 \mathrm{eV}$ на атомах углерода графана с шириной запрещенной зоны $\sim 5 \mathrm{eV}$ (см. рис. 1, $a$ в [9]). Численный расчет показал, что уровни бивакансии лежат на расстояниях, равных приблизительно $\pm 1.5 \mathrm{eV}$ от центра запрещенной зоны (см. рис. 2, с в [9]). 


\section{4. Фононные частоты и упругие постоянные}

Для оценок фононных характеристик графана воспользуемся методом связывающих орбиталей Харрисона $[25,26]$. Энергию $E_{b}$ связи $\mathrm{C}-\mathrm{H}$ и соответствующую силовую константу центрального взаимодействия $k_{0 \perp}$ можно представить в виде

$$
\begin{gathered}
E_{b}=2 V_{2} \alpha_{c}^{-1}\left(1-2 \alpha_{c} / 3\right), \\
k_{0 \perp}=4 \alpha_{c} V_{2}\left(1-2 \alpha_{p}^{2}\right) / a_{\perp}^{2},
\end{gathered}
$$

где $V_{2}-$ ковалентная энергия, $\alpha=V_{2} / \sqrt{V_{2}^{2}+V_{3}^{2}}$ и $\alpha_{p}=\sqrt{1-\alpha_{c}^{2}}-$ ковалентность и полярность связи $\mathrm{C}-\mathrm{H}$, длина которой равна $\alpha_{\perp}=1.11 \AA, V_{3}-$ полярная энергия (см. подробнее [25-27]). Полагая, что имеет место $\sigma$-связь $s$-орбитали атома $\mathrm{H}$ с $p$-орбиталью атома С, имеем $V_{2}=V_{s p \sigma}=\eta_{s p \sigma}\left(\hbar^{2} / m_{0} a_{\perp}^{2}\right)$, где $\eta_{s p \sigma}=1.42[26]$, так что $V_{2}=8.78 \mathrm{eV}$. Полярная энергия $V_{3}=\left(\varepsilon_{c p}-\varepsilon_{h s}\right) / 2$, где $\varepsilon_{c p}=-11.07 \mathrm{eV}$ есть энергия $p$-состояния атома $\mathrm{C}$ (использованы таблицы атомных термов Манна [28]) и $\varepsilon_{h s}=-13.60 \mathrm{eV}-$ энергия $s$-состояния атома $\mathrm{H}$ (взятый с обратным знаком потенциал ионизации) равна $V_{3}=0.765 \mathrm{eV}$, так что $\alpha_{c} \approx 0.996$ и $\alpha_{p} \approx 0.087$. Полагая $\alpha_{c}=1$ и $\alpha_{P}=0$, из выражения (28) получим $E_{b} \approx 2 V_{2} / 3=5.85 \mathrm{eV}$, тогда как расчет [3] дает $4.79 \mathrm{eV}$. Силовая константа $k_{0 \perp} \approx 4 V_{2} / a_{\perp}^{2}=28.5 \mathrm{eV} / \AA$, а соответствующая частота $\Omega_{\perp}=\sqrt{k_{0 \perp} / m_{p}}\left(m_{p}\right.$ - масса протона) равна $0.344 \mathrm{eV}$, или $2774 \mathrm{~cm}^{-1}$. Эта частота на $145 \mathrm{~cm}^{-1}$ ниже вычисленного из первых принципов значения $\Omega_{\perp}=2919 \mathrm{~cm}^{-1}[1]$ (практически тот же результат для $\Omega_{\perp}$ получен в расчетах [3]). Отметим, что при оценке $\Omega_{\perp}$ мы учитывали, что атом водорода связан не со свободным атомом углерода, а с атомом, находящимся в узле графенового листа. Поэтому формально можно положить $m_{p} \ll M_{C}^{*}$, где $M_{C}^{*}$ — „эффективная масса“ атома углерода графана, т. е. атом водорода привязан к неподвижному листу.

Если, как в работах $[1,3]$, считать, что $\sigma$-связь атомов С и Н осуществляется соответственно $s p^{3}$ и $s$-орбиталями, то $V_{2}=V_{s / s p 3}=\eta_{s / s p 3}\left(\hbar^{2} / m_{0} a_{\perp}^{2}\right)$, где $\eta_{s / s p 3}=1.89$ [29], получим $V_{2}=11.69 \mathrm{eV}$. Энергия $s p^{3}$-орбитали атома углерода $\varepsilon_{c s p 3}=\left(\varepsilon_{c s}+3 \varepsilon_{c p}\right) / 4$ $=-13.15 \mathrm{eV}$ (таблицы Манна), так что $V_{3}=0.225 \mathrm{eV}$. Поэтому вновь можно положить $\alpha_{c}=1$ и $\alpha_{p}=0$. Получим, таким образом, $k_{0 \perp}=38.6 \mathrm{eV} / \AA, \Omega_{\perp}=0.4 \mathrm{eV}$, или $3230 \mathrm{~cm}^{-1}$, что на $311 \mathrm{~cm}^{-1}$ значительно выше $\Omega_{\perp}=2919 \mathrm{~cm}^{-1}$. При этом, подчеркнем, что оценки частоты $\Omega_{\perp}$ для $p-s$-связи ближе к результатам $[1,3]$, чем для связи $s p^{3}-s$. Следует отметить также, что центральная силовая константа $k_{0 \perp}$ описывает реакцию графана на сжатие или растяжение связи в направлении, перпендикулярном листу, и поэтому является одномерным аналогом объемного модуля сжатия.
Оценим теперь характерную энергию электронфононной связи $\lambda=w^{2} / k_{0 \perp}$, где деформационный фактор $w=-\partial V_{2} / \partial a_{\perp}$ [30]. Так как $-\partial V_{2} / \partial a_{\perp}=2 V_{2} / a_{\perp}$ и $k_{0 \perp} \approx 4 V_{2} / a_{\perp}^{2}$, получим $\lambda \approx V_{2}$. Эта величина гораздо выше, чем значения $\lambda$ для адсорбированных на графене двух-, трех- и четырехатомных молекул газа, адсорбированных на графене [30], Так как $\lambda \propto a_{\perp}^{-2}$, такой результат объясняется малой длиной связи $\mathrm{C}-\overrightarrow{\mathrm{H}}$.

Перейдем к колебаниям в плоскости листа графана. При этом допустимо, на наш взгляд, считать, что атомы водорода жестко связаны с соответствующими атомами углерода, так что смещаются вместе с ними без какой-либо деформации связей С-H. Таким образом, отличия от соответствующих колебаний в плоскости листа графена лишь количественные: масса атомов углерода увеличивается на одну протонную массу и силовые константы уменьшаются вследствие увеличения межатомного расстояния в графане $a=1.52 \AA$ по сравнению с межатомным расстоянием в графене $\bar{a}=1.42 \AA$. Тогда для центральных силовых констант отношение $k_{0} / \bar{k}_{0}=(\bar{a} / a)^{4} \approx 0.76$, если предполагать, что $\sigma$-связи в листах графена и графана образованы орбиталями с одним и тем же типом гибридизации. Тогда $\Omega / \bar{\Omega}=(\bar{a} / a)^{2} \sqrt{12 / 13} \approx 0.84$. Если же считать, что в случае графена имеет место $s p^{2}$-гибридизация, а для графана $s p^{3}$-гибридизация, то $k_{0} / \bar{k}_{0}=\left(\eta_{s p 3} / \eta_{s p 2}\right)(\bar{a} / a)^{4} \approx 0.75$ и $\Omega / \bar{\Omega}=(\bar{a} / a)^{2} \sqrt{(12 / 13)\left(\eta_{s p 3} / \eta_{s p 2}\right)} \approx 0.83$, где $\eta_{s p k}$ - коэффициенты пропорциональности для матричных элементов $V_{s p k}: \eta_{s p 2}=3.26$ и $\eta_{s p 3}=3.22$ [26]. Так как частота длинноволновых (соответствующих точке Г) оптических колебаний атомов углерода свободного графена равна $1580 \mathrm{~cm}^{-1}$ ( $G$-пик раман-спектра) [31], для графана получим $1325 \mathrm{~cm}^{-1}$ при $s p^{2}$-гибридизации и $1317 \mathrm{~cm}^{-1}$ при $s p^{3}$-гибридизации. Такие значения частот хорошо согласуется с результатами численных расчетов $[1,3,32]$. Подчеркнем, что различие $s p^{2}$ - и $s p^{3}$-гибридизаций играет здесь крайне малозначительную роль. Отметим также следующее: расчет [33] показал, что модуль всестороннего сжатия графана на $26 \%$ меньше, чем у графена, что отлично согласуется с нашей оценкой отношения $k_{0} / \bar{k}_{0}$. Сходные результаты дают расчеты [34,35].

В работе [36] в рамках модели Китинга [37] нами были получены следующие выражения для упругих постоянных графена $c_{11}$ и $c_{12}$, описывающих реакцию на деформацию в плоскости листа

$$
\begin{aligned}
& c_{11}=\frac{1}{\sqrt{3}}\left(4 \alpha+\beta+18 \frac{\alpha \beta}{4 \alpha+\beta}\right), \\
& c_{12}=\frac{1}{\sqrt{3}}\left(4 \alpha+\beta-18 \frac{\alpha \beta}{4 \alpha+\beta}\right),
\end{aligned}
$$

где $\alpha$ и $\beta-$ силовые константы центрального и нецентрального взаимодействий, значения которых определим из рассчитанных в [35] модуля Юнга $E=\left(c_{11}^{2}-c_{12}^{2}\right) / c_{11}=246.7 \mathrm{~N} / \mathrm{m}$ и коэффициента Пуассона $\sigma=c_{12} / c_{11}=0.078$ (или $c_{11}=248.2 \mathrm{~N} / \mathrm{m}$ 
и $\left.c_{12}=19.4 \mathrm{~N} / \mathrm{m}\right)$. Вновь полагая, что атомы водорода в процессе плоской деформации остаются жестко связанными с атомами углерода, из (30) получаем $\alpha=43 \mathrm{~N} / \mathrm{m}$ и $\beta=59.3 \mathrm{~N} / \mathrm{m}$. Полученный результат интересен тем, что отношение $\beta / \alpha>1$. Действительно, для графена $\alpha=77 \mathrm{~N} / \mathrm{m}$ и $\beta=69 \mathrm{~N} / \mathrm{m}$ [27] (при этом использовались значения $E=340 \pm 50 \mathrm{~N} / \mathrm{m}$ и $\sigma=0.165$ [38]). Более того, для трехмерных полупроводников III-V II-VI [36,39,40] отношение $\beta / \alpha<1$, причем с ростом полярности связи убывает.

\section{5. Заключение}

В настоящей работе мы получили аналитические оценки ряда электронных характеристик идеального и дефектного графана. При этом для определения параметров мы использовали результаты имеющихся численных расчетов. Предложенная параболическая аппроксимация электронного спектра позволила предсказать значения эффективной массы носителей и квантовой емкости. Оценки характерных фононных частот выполнены в рамках метода связывающих орбиталей Харрисона без использования каких-либо подгоночных параметров. Полученные результаты представляются вполне разумными. К сожалению, экспериментальная информация по графану практически отсутствует, не известно даже реальное значение ширины запрещенной зоны. По поводу перспектив исследования свойств графана и его применений см. краткие обзоры $[32,41]$.

\section{Конфликт интересов}

Автор заявляет об отсутствии конфликта интересов.

\section{Список литературы}

[1] J.O. Sofo, A.S. Chaudhari, G.D. Barber. Phys. Rev. B 75, 153401 (2007).

[2] D.C. Elias, R.R. Nair, T.M.G. Mohiuddin, S.V. Morozov, P. Blake, M.P. Halsall, A.C. Ferrari, D.W. Boukhvalov, M.I. Katsnelson, A.K. Geim, K.S. Novoselov. Science 323, 610 (2009).

[3] H. Şahin, C. Ataca, S. Ciraci. Appl. Phys. Lett. 95, 222510 (2009).

[4] S. Lebégue, M. Klintenberg, O. Eriksson, M.I. Katsnelson. Phys. Rev. B 79, 245117 (2009).

[5] D.W. Boukhvalov, M.I. Katsnelson, A.I. Lichtenstein. Phys. Rev. B 77, 35427 (2008).

[6] M.R. Thomsen, M.M. Ervasti, A. Harju, T.G. Pedersen. Phys. Rev. B 92, 195408 (2015).

[7] H.-C. Huang, S.-Y. Lin, C.-L. Wu, M.-F. Lin. Carbon 103, 84 (2016).

[8] H.-Y. Lu, L. Hao, R. Wang, C.S. Ting. Phys. Rev. B 93, 241410(R) (2016).

[9] L. Hao, H.-Yan Lu, C.S. Ting. Phys. Rev. Mater. 3, 024003 (2019). arXiv: $1812.0777 \mathrm{v} 1$.

[10] С.Ю. Давыдов. ФТТ 58, 779 (2016).
[11] T.O. Wehling, E. Sasioglu, C. Friedrich, A.I. Lichtenstein, M.I. Katsnelson, S. Blügel. Phys. Rev. Lett. 106, 236805 (2011).

[12] T. Ando, A.B. Fowler, F. Stern. Rev. Mod. Phys. 54, 437 (1982).

[13] S. Luryi. Appl. Phys. Lett. 96, 501 (1988).

[14] D.L. John, L.C. Castro, D.L. Pulfrey. J. Appl. Phys. 96, 5180 (2004).

[15] С.Ю. Давыдов, А.А. Лебедев, П.В. Булат, А.В. Зубов. Письма в ЖТФ 46, 15, 7 (2020).

[16] Дж. Слетэр. Диэлектрики, полупроводники, металлы. Мир, М. 1969.

[17] Дж. Каллуэй. Теория энергетической зонной структуры. Мир, М. 1969.

[18] С.Ю. Давыдов, А.А. Лебедев, О.В. Посредник. Элементарное введение в теорию наносистем. Изд-во „Лань“, СПб (2014).

[19] С.Ю. Давыдов. ФТП 50, 816 (2016).

[20] С.Ю. Давыдов, А.В. Зубов. ФТП 54, 170 (2020).

[21] F.D.M. Haldane, P.W. Anderson. Phys. Rev. B 6, 2553 (1976).

[22] С.Ю. Давыдов, С.В. Трошин. ФТТ 50, 1206 (2008).

[23] С.Ю. Давыдов. ФТП 46, 204 (2012).

[24] С.Ю. Давыдов. ФТТ 54, 2193 (2012).

[25] У. Харрисон. Электронная структура и свойства твердых тел. Мир, М. (1983).

[26] W.A. Harrison. Phys. Rev. B 27, 3592 (1983).

[27] С.Ю. Давыдов, О.В. Посредник. ФТТ 57, 819 (2015).

[28] W.A. Harrison. Phys. Rev. B 31, 2121 (1985).

[29] С.Ю. Давыдов, Г.И. Сабирова. Письма в ЖТФ 37, 11, 51 (2011).

[30] С.Ю. Давыдов. Письма в ЖТФ 44, 3, 40 (2018).

[31] Z.H. Ni, W. Chen, X.F. Fan, J.L. Kuo, T. Yu, A.T.S. Wee, Z.X. Shen. Phys. Rev. B 77, 115416 (2008).

[32] H. Sahin, O. Leenaerts, S.K. Singh, F.M. Peeters. arXiv: $1502.05804 \mathrm{v} 1$.

[33] K. Xue, Z. Xu. Appl. Phys. Lett. 96, 063103 (2010).

[34] E. Muñoz, A.K. Singh, M.A. Ribas, E.S. Penev, B.I. Yakobson. Diamond Rel. Mater. 19, 368 (2010).

[35] Q. Peng, C. Liang, W. Ji, S. De. Phys. Chem. Chem. Phys. 15, 2003 (2013).

[36] С.Ю. Давыдов. ФТТ 52, 756 (2010).

[37] P.N. Keating. Phys. Rev. 145, 637 (1965).

[38] C. Lee, X. Wei, J.W. Kysar, J. Hone. Science 321, 385 (2008).

[39] R.M. Martin. Phys. Rev. B 1, 4005 (1970).

[40] С.Ю. Давыдов, А.В. Соломонов. Письма в ЖТФ 25, 15, 23 (1999).

[41] C. Zhou, S. Chen, J. Lou, J. Wang, Q. Yang, C. Liu, D. Huang, T. Zhu. Nanoscale Res. Lett. 9, 26 (2014). http://www.nanoscalereslett.com/content/9/1/26

Редактор Т.Н. Василевская 